\title{
Research of Reliability Assessment of Air Traffic Management Automation Equipment
}

\author{
Weijun PAN ${ }^{1, \text { a }}$, Yuexiao PAN ${ }^{2, b^{*}}$, Zifeng $\mathrm{ZHOU}^{3, \mathrm{c}}$, Jingwei TAN ${ }^{4, \mathrm{~d}}$ \\ ${ }^{1}$ Civil Aviation Flight University of China, Guanghan Sichuan, 618300, China \\ ${ }^{2}$ Civil Aviation Flight University of China, Guanghan Sichuan, 618300, China \\ ${ }^{3}$ Civil Aviation Flight University of China, Guanghan Sichuan, 618300, China \\ ${ }^{4}$ Civil Aviation Flight University of China, Guanghan Sichuan, 618300, China \\ Email: panyuexiao2010@163.com
}

\begin{abstract}
Key words: Control equipment; Reliability level; Fuzzy analysis; Analytic hierarchy process; Evaluation model.

Abstract. To solve the issue that there is some lack of subjectivity and objectivity in the Traditional reliability assessment of air traffic management evaluation means and by analyzing and finding out the related and essential factors in the reliability assessment process of air traffic management equipment, a fuzzy level comprehensive evaluation model can be established and reliability levels of the control equipment are Quantized .SoW that the reliability and evaluation credibility of the equipment are improved. According to the examples below, it turns out that the solution referred can reduce the subject bias in the assessment process, so the result of assessment is more logical, scientific and normative relatively.
\end{abstract}

\section{Introduction}

Equipment risk assessment is an important mean to provide bases to equipment life cycle management and the planning and management of its support services. Air traffic management equipment is a complex system, so the risks are related to many factors, including the error, repairing and spare equipping of the equipment, running environment, maintenance personnel and managing regulations, etc. Traditional evaluation means are limited due to the randomness and fuzziness of these factors, so the evaluating means existing are mainly depended on evaluators' experiences, instead of a united, scientific, reasonable and normative standard.

Fuzzy synthetic discrimination provides a scientific solution to systematically analyze problem and get quantitative and qualitative ways together, which can recover fuzzy concept of evaluation target better, so the consequence has rather-high reliability, logicality and standardization. As a result, by accurately analyzing the key factors influencing comprehensive support capability to equipment of ATC (Air Traffic Control), an effectiveness evaluation model basing on fuzzy mathematics and analytic hierarchy process can be established to evaluate support capacity of ATC equipment [1,2].

\section{Reliability analysis index}

Reliability is an important part of subject of reliability. In 2070th, the subject of reliability paced into machine equipment and products in a rather-high speed in industrial-developed countries. In 2080th, China started to pay attention to the reliability research of equipment, but there was little base because of the late starting time. Normally, important index of equipment to evaluate reliability are as follow: no fault resistance, maintainability, availability, durability, performance retention, economy and security, etc. The choice of reliability index should obey the regulations as follows:

(1) Integrity. Which means it can fully reflect the reliability of equipment, focusing on comprehension, accuracy, reliability and effectiveness.

(2) Convenience. Which means the data can be estimated easily from experiment data or running equipment. 
(3) Comparability. A united standard of calculating and evaluating should be established in similar products to compare similar products between different companies more easily.

(4) Progressiveness. The standard adapted should be international, so that it would be easier to compare products with companies domestic and abroad.

\section{Establishment of the fuzzy relationship matrix}

The basic principle of fuzzy evaluation is : The factor set of object $\mathrm{C}=\left(c_{1}, c_{2}, \ldots, c_{n}\right)$ and the evaluation set on $V=\left(v_{1}, v_{2} \ldots, v_{n}\right)$ is determined firstly. $u_{i}$ is various index, $v_{j}$ is judging hierarchy to $u_{i}$. Then ensure the weight of each factor $M$ and their membership degree vector R. Get Fuzzy evaluation matrix after fuzzy transformation. Finally, the fuzzy evaluation matrix and the factor of the weight vector will be normalized. Get evaluation set on $\mathrm{S}, S=M * R$, so $(\mathrm{c}, \mathrm{v}, \mathrm{r}, \mathrm{m})$ constitutes a judge set model[3].

\section{Determination of the fuzzy relationship matrix}

After constructing the fuzzy sets, we should make a quantitative evaluation to each factor, and determine the membership degree of the fuzzy subset from the single factor. The single factor comprehensive evaluation relation matrix can be established as follows [4]:

$$
\boldsymbol{R}=\left[\begin{array}{rrrr}
r_{11} & r_{22} & \ldots & r_{1 m} \\
r_{21} & r_{22} & \ldots & r_{2 m} \\
\ldots & \ldots & \ldots & \ldots \\
r_{n 1}^{r} & r_{n 2} & \ldots & r_{n m}
\end{array}\right]=\left(r_{i j}\right)_{n m}
$$

In the formula (1), $\boldsymbol{R}$ is the comments fuzzy vector about factor $c_{i} ; \boldsymbol{R}$ is the membershipNo. $i$ factor's assessment to the No.j level, which means $u_{i}$ 's extent of having $v_{j}$ comments.

\section{Weight coefficient matrix}

According to the statistical methods or experts rate methods, for example, weight distribution between the factors can be established to make factors have a same united balance. Weight coefficient matrix can be shown as follow:

$$
\boldsymbol{m}=\left(m_{1}, m_{2}, \ldots, m_{i}\right), m_{i} \geq 0 \text {, and } \sum_{i=1}^{m} m_{i}=1
$$

Based on that, we can apply $A H P$ to ensure the weights of factors. This solution can resolute complex and many factors, make up an ordered hierarchical structure, and ensure the weights of index by comparing each other and calculating matrix's largest eigenvalue and its corresponding eigenvector [5].

(1) Judge matrix establishing

For comparing the influence of every factor to the target and ensure the importance of the factors to the target, analytic hierarchy process set $A_{i j}$ the ratio of the impact of two factors on target, and set $\boldsymbol{A}$ the result matrix of all the ratios. 1 to 9 scale method comparison is commonly used, which can be got from experts or technical security sector personnel, according to the nature of each indicator, the relative relationship and practical experience, as shown in Table 1.

Table 1 Scale and Description

\begin{tabular}{cc}
\hline Scale & Definition and Description \\
\hline 1 & Two elements have same importance to certain property \\
4 & Compared, one element is little more important than the other \\
7 & Compared, one element is rather more important than the other \\
9 & Compared, one element is obviously more important than the other \\
$2,4,6,8$ & Compared, one element is extremely more important than the other \\
$1 / A_{i j}$ & Indicates the scale between the standards before \\
\hline
\end{tabular}

If there is a judge matrix, $A=\left[a_{i j}\right]_{n+m}$, in which $n$ is the dimension of the judge matrix, the elements in the judge matrix should obey: 


$$
a_{i j}=\frac{1}{a_{i j}} * a_{i i}=1
$$

(2) Hierarchical order

According to the judge matrix, the eigenvector corresponding to the maximum eigenvalue can be calculated, which also reflects the order of importance of each evaluation factor that also means weights. In the situation here, the eigenvalues of $\boldsymbol{A}$ and their eigenvectors should be calculated, in which the eigenvector $\boldsymbol{m}$ responding to the maximum eigenvalue $\lambda_{\max }$ is the weights required.

(3) Consistency check

The weights get above is whether reasonable or not, should be judged by the consistency check of the matrix. The formula is $C R=\frac{C I}{R I}$, in which $C I=\left(\frac{A_{\max }-n}{n-1}\right)$ and $R I$ is called as the mean random consistency index of judgment matrix. As for the matrix of 1 to 9 dimensions [6], $R I$ is shown in Table 2.

Table $2 R I$ of judge matrixes of 1 to 9 dimensions

\begin{tabular}{cccccccccc}
\hline$n$ & 1 & 2 & 3 & 4 & 5 & 6 & 7 & 8 & 9 \\
$R I$ & 0 & 0 & 0.58 & 0.90 & 1.12 & 1.24 & 1.32 & 1.41 & 1.45 \\
\hline
\end{tabular}

When $C R<0.1$, the judge matrix has satisfying consistency, which means weights are reasonable, or the judge matrix should be adjusted until it has satisfying consistency.

\section{Fuzzy comprehensive assessment models}

According to the complex operation rules of fuzzy mathematics matrix, the main risk fuzzy comprehensive evaluation matrix can be calculated:

$$
S=M R=\left(m_{1}, m_{2}, \ldots, m_{n}\right) \times\left[\begin{array}{cccc}
r_{11} & r_{12} & \ldots & \boldsymbol{r}_{1 m} \\
r_{21} & r_{22} & \ldots & \mathbf{r}_{2 m} \\
\ldots & \ldots & \ldots & \ldots \\
r_{n 1} & r_{n 2} & \ldots & \boldsymbol{r}_{m m}
\end{array}\right]
$$

In the formula (4), $S$ is the fuzzy set on $V, S=\left(S_{1}, S_{2}, \ldots, S_{n}\right)$;

\section{The application of overview in reliability analysis of control equipment}

Five index, including equipment failure rate, MTBF (Mean Time Between Failures), fault monitoring level, equipment maintenance time needed and capable accommodate control service levels, etc., can be applied as assessment index to have reliability analysis on a certain domestic ATC equipment and construct the structure model following, express this indexes in terms of $n 1, n 2, n 3, n 4, n 5$.

\section{Applying example}

By collecting historical running data of a certain domestic ATC center and understanding ATC equipment, we can calculate weights by the method mentioned before.

The eigenvalue of judge matrix :

$$
\lambda_{\max }=5.27, C I=\frac{\lambda_{\max }-5}{5-1}=0.0675, C R=\frac{C l}{1.12}=0.06<0.1 \text {; }
$$

So the consistency is satisfied. The result is shown as Table 3. 
Table 3 The judge matrix between evaluation criteria of ATC automation system

\begin{tabular}{ccccccc}
\hline Rules & $n_{1}$ & $n_{2}$ & $n_{3}$ & $n_{4}$ & $n_{5}$ & $m_{i}$ \\
\hline$n_{1}$ & 1 & 2 & $1 / 3$ & 2 & $1 / 7$ & 0.0936 \\
$n_{2}$ & $1 / 2$ & 1 & $1 / 2$ & $1 / 2$ & $1 / 8$ & 0.0594 \\
$n_{3}$ & 3 & 2 & 1 & 5 & $1 / 4$ & 0.2038 \\
$n_{4}$ & $1 / 2$ & 2 & $1 / 5$ & 1 & $1 / 8$ & 0.0652 \\
$n_{5}$ & 7 & 8 & 4 & 8 & 1 & 0.5779 \\
\hline
\end{tabular}

The situation of the ATC equipment can be get by expert scoring method, in which there are 10 experts scoring in 5 grades, as is shown in Table 4.

Table 4 Expert score set

\begin{tabular}{ccccc}
\hline Factor Set & $v_{1}$ (excellent) & $v_{2}$ (good) & $v_{3}(\mathrm{OK})$ & $v_{4}$ (worst) \\
\hline$n_{1}$ & 8 & 2 & 0 & 0 \\
$n_{2}$ & 4 & 5 & 1 & 0 \\
$n_{3}$ & 5 & 5 & 0 & 0 \\
$n_{4}$ & 7 & 3 & 0 & 0 \\
$n_{5}$ & 6 & 1 & 2 & 1 \\
\hline
\end{tabular}

Let $r_{i j}=\frac{c_{i j}}{\Sigma_{j=1}^{5} c_{i j}}$, in which $\sum_{j=1}^{5} C_{i j}=10$ means the amount of experts.

Single factor evaluation matrix can be calculated from the formula above:

$$
\boldsymbol{R}=\left[\begin{array}{cccc}
0.8 & 0.2 & 0 & 0 \\
0.4 & 0.5 & 0.1 & 0 \\
0.5 & 0.5 & 0 & 0 \\
0.7 & 0.3 & 0 & 0 \\
0.6 & 0.1 & 0.2 & 0.1
\end{array}\right]
$$

Comprehensive Evaluation of Fuzzy Analytic Hierarchy Process:

$$
\boldsymbol{S}=\boldsymbol{M} * \boldsymbol{R}=\left[\begin{array}{llll}
0.5919 & 0.2270 & 0.1215 & 0.0578
\end{array}\right]
$$

According to the maximum membership degree principle, the reliability of ATC equipment can be judged as excellent, which means the ATC automation equipment has high reliability level and meets the need of ensuring daily flight safety.

\section{Summary}

In the reliability assessment of ATC equipment, it is important to set the appraisal program and scoring method scientifically and reasonably. The fuzzy complex evaluating model should reflect the relationship between each index quantitatively and systematically, so that the reliability can be objectively reflected and scientific accordance can be getting for higher reliability of the equipment. Besides, the test and evaluating method of the reliability of ATC equipment is simply discussed in this article, and the fuzzy complex Assessment is applied to the area of ATC equipment reliability testing, in which assessment index system and choice regulations are given out. In addition, the assessment model is established while the weights calculation method is obtained. Finally, we demonstrate the feasibility by the fuzzy comprehensive evaluation example.

\section{Acknowledgement}

This work is supported by the National ATC Research Found (GKG201403001), Airport capacity management and time demand forecasting management system (XN2013003). 


\section{References}

[1] Yuan Shangnan, Qiang Maoshan, Wen Qi, Jiang Hanchen. Evaluation model of organizational construction projects based on Fuzzy Analytic Hierarchy Process [J] Journal of Tsinghua University (natural science edition) 2015, 55(6): 616-623.

[2] Wang Yu,Wang Yongchao, Niu Yinbao, Zhao Jianping The Optimal selection of Numerical control machine tool equipment based on Fuzzy analytic hierarchy process $[\mathrm{J}]$.Combination machine tools and processing technology Automated, 2014(11): 133-136.

[3] Wei Kai, evaluation of construction project investment risk based on fuzzy analytic hierarchy process method [J], International Conference on Advanced Management Science, v 2, p 10-14, 2010.

[4] Sotoodeh Gohar,A. Construction projects risk assessment based on fuzzy AHP[J], SCOReD2009 Proceedings of 2009.

[5] Robert K.Wysocki,Robert Beck Jr,Effective Project Management(2nd Edition)[M],UK,John Wiley\&Sons, 2007.

[6] Wang Xinmin,Zhao Bin, Zhang Qinli. Selection of mining method based on analytic hierarchy process and fuzzy mathematics [J]. Journal of Central South University: Natural Science Edition, 2008, 39 (5): 875 880. 